EPJ Web of Conferences 113, 04020 (2016)

DOI: $10.1051 /$ epjconf/201611304020

C) Owned by the authors, published by EDP Sciences, 2016

\title{
Chiral nuclear forces: Recent developments
}

\author{
Evgeny Epelbaum ${ }^{1, a}$ \\ ${ }^{1}$ Institut für Theoretische Physik II, Ruhr-Universität Bochum, D-44780 Bochum, Germany
}

\begin{abstract}
In this talk, I review recent progress towards developing accurate and precise nuclear forces in the framework of chiral effective field theory. The new generation of NN potentials up to fifth order in the chiral expansion is shown to provide excellent description of phase shifts and to yield a convergent pattern for low-energy NN observables. I also address the issue of uncertainty quantification and discuss some recent results in nucleon-deuteron scattering.
\end{abstract}

\section{Introduction}

Chiral effective field theory (EFT) provides a well established and nowadays most commonly used approach to low-energy nuclear reactions and light nuclei, see [1,2] for recent review articles. It relies on the symmetries of QCD and allows one to derive nuclear forces and the corresponding current operators within a systematically improvable perturbative expansion, the so-called chiral expansion [3]. In the standard formulation based on pions and nucleons as the only explicit degrees of freedom, the expansion parameter $Q$ is defined as $Q \in\left\{M_{\pi} / \Lambda_{b},|\vec{p}| / \Lambda_{b}\right\}$ with $M_{\pi}, \vec{p}$ and $\Lambda_{b}$ referring to the pion mass, typical three-momenta of the nucleons and the breakdown scale to be specified below, respectively.

In the past two and a half decades, this approach was employed by different groups to derive nuclear forces up to fourth order $Q^{4}$ in the chiral expansion $\left(\mathrm{N}^{3} \mathrm{LO}\right)$ [4-8] and to perform ab initio calculations of few-nucleon reactions and light nuclei. For a related recent work on the electromagnetic currents see [9-12]. Given that the numerical implementation of the $\mathrm{N}^{3} \mathrm{LO}$ three-nucleon force (3NF) contributions represents a non-trivial task, which could be accomplished only recently [13, 14], most of the available $a b$ initio calculations utilized the nuclear Hamiltonian consisting of the $\mathrm{N}^{3} \mathrm{LO}$ nucleon-nucleon $(\mathrm{NN})$ potentials of $[4,5]$ accompanied by the leading, i.e. order- $Q^{3}\left(\mathrm{~N}^{2} \mathrm{LO}\right), 3 \mathrm{NF}$. These studies have revealed the important role of the chiral 3NF yielding many promising results, see [15] and references therein. For a progress report on the discretized formulation of nuclear chiral EFT see [16]. Still, the well-known discrepancies between state-of-the-art theoretical calculations and experimental data in the three-nucleon continuum at intermediate energies of $E_{N} \sim 50 \ldots 100 \mathrm{MeV}$ and higher [17] could so far not be resolved. Further developments are needed to tackle the long-standing $3 \mathrm{NF}$ problem in the framework of chiral EFT. First, given that the $3 \mathrm{NF}$ is itself a small correction to the dominant $\mathrm{NN}$ force, accurate and precise chiral NN potentials are required to pin down the $3 \mathrm{NF}$ effects. Secondly, it was argued that the chiral expansion of the $3 \mathrm{NF}$ is not yet converged at $\mathrm{N}^{3} \mathrm{LO}$ [18] and should, therefore, be pushed to at least $\mathrm{N}^{4} \mathrm{LO}$ (i.e. $Q^{5}$ ) in the standard approach [19-21] or

\footnotetext{
a e-mail: evgeny.epelbaum@rub.de
} 
to $\mathrm{N}^{3} \mathrm{LO}$ in the formulation with explicit $\Delta(1232)$ degrees of freedom. Last but not least, it is crucial to be able to reliably quantify the theoretical uncertainty due to truncation of the chiral expansion for all calculated observables, see [22] for a general discussion. In this talk I discuss recent developments along these lines focusing mainly on the first and the last topics.

\section{A new generation of chiral $\mathrm{NN}$ forces up to $\mathrm{N}^{4} \mathrm{LO}$}

Given that the chiral expansion of the $3 \mathrm{NF}$ is not yet converged at $\mathrm{N}^{3} \mathrm{LO}$, the expansion for the nuclear Hamiltonian needs to be pushed to $\mathrm{N}^{4} \mathrm{LO}$. For the $\mathrm{NN}$ force, the pertinent long-range contributions due to $2 \pi$ - and $3 \pi$-exchange have been derived and applied to peripheral NN scattering in Ref. [23]. Notice that no new isospin-conserving $\mathrm{NN}$ contact interactions appear at this order in the chiral expansion.

In $[24,25]$, we have introduced a new generation of $\mathrm{NN}$ potentials up to $\mathrm{N}^{4} \mathrm{LO}$ in the chiral expansion. In addition to pushing the chiral expansion to a higher order, these new potentials feature a number of important improvements as compared to the first-generation chiral $\mathrm{N}^{3} \mathrm{LO} \mathrm{NN}$ forces. First, we used a coordinate-space regularization procedure for the long-range components $V_{\text {long }}$,

$$
V_{\text {long }}(\vec{r}) \rightarrow V_{\text {long }}^{\text {Reg. }}(\vec{r})=V_{\text {long }}(\vec{r}) F\left(\frac{r}{R}\right) \quad \text { with } \quad F(x)=\left(1-\exp \left(-x^{2}\right)\right)^{6},
$$

where the short-range cutoff $R$ is chosen in the range of $R=0.8 \ldots 1.2 \mathrm{fm}$ in agreement with the estimated breakdown distance of $\sim 0.8 \mathrm{fm}$ of the chiral expansion for the pion-exchange potential [26]. The new regulator maintains, per construction, the analytic structure of the amplitude at low momenta and allowed us to substantially reduce the amount of finite-cutoff artefacts. Moreover, since the new regulator cuts off precisely the undesired short-range components of the pion exchange contributions instead of their large-momentum parts as the old non-local regulator does, no additional spectralfunction regularization (SFR) [27] used e.g. in [4] is required. This is a particularly welcome feature in view of the ongoing and upcoming 3NF studies, in which the implementation of the SFR would be highly non-trivial. Notice that while the authors of [5] have managed to avoid the introduction of the SFR at $\mathrm{N}^{3} \mathrm{LO}$, this was achieved at the cost of employing a nonlocal regulator, whose functional form was adjusted from partial wave to partial wave, see Table F.2 of [2].

Secondly, the new potentials of $[24,25]$ adopt the values of the $\pi N$ low-energy constants (LECs) determined in $\pi N$ scattering without any fine tuning. In particular, at $\mathrm{N}^{4} \mathrm{LO}$, we used the LECs taken from the order- $Q^{4}$ fit to the Karlsruhe-Helsinki partial wave analysis (PWA) of [19], see [28] for a recent extraction from the subthreshold parameters and [29] for a related work.

With the short-range contact interactions being determined from a fit to the Nijmegen PWA (NPWA) of neutron-proton (np) and proton-proton (pp) scattering [30], see [24, 25] for more details, our results for S-, P- and D-waves and the mixing angles $\epsilon_{1,2}$ at different chiral orders are visualized in Fig. 1 for $R=0.9 \mathrm{fm}$. One observes a very good convergence pattern of the chiral expansion in the whole energy range up to $E_{\text {lab }}=300 \mathrm{MeV}$. To get more quantitative insights, we show in Table 1 the $\chi^{2} /$ datum for the description of the Nijmegen $\mathrm{np}$ and pp phase shifts. Here, we estimated the uncertainty of the NPWA for a given phase shift and mixing angle from the deviations to the results of the Nijmegen I, II and Reid93 potentials [31], which are regarded as alternative PWA. Notice, however, that such an estimation does not allow for a statistical interpretation of the $\chi^{2}$ values shown in table 1 . Still, the $\chi^{2}$ defined as described above is a useful tool to quantify the accuracy of the fits.

As expected, one observes the improved description of the phase shifts with increasing orders of the chiral expansion. It is particularly encouraging to see a strong reduction in the $\chi^{2}$ when going from $\mathrm{NLO}\left(Q^{2}\right)$ to $\mathrm{N}^{2} \mathrm{LO}\left(Q^{3}\right)$ and from $\mathrm{N}^{3} \mathrm{LO}\left(Q^{4}\right)$ to $\mathrm{N}^{4} \mathrm{LO}\left(Q^{5}\right)$, which comes entirely from the 

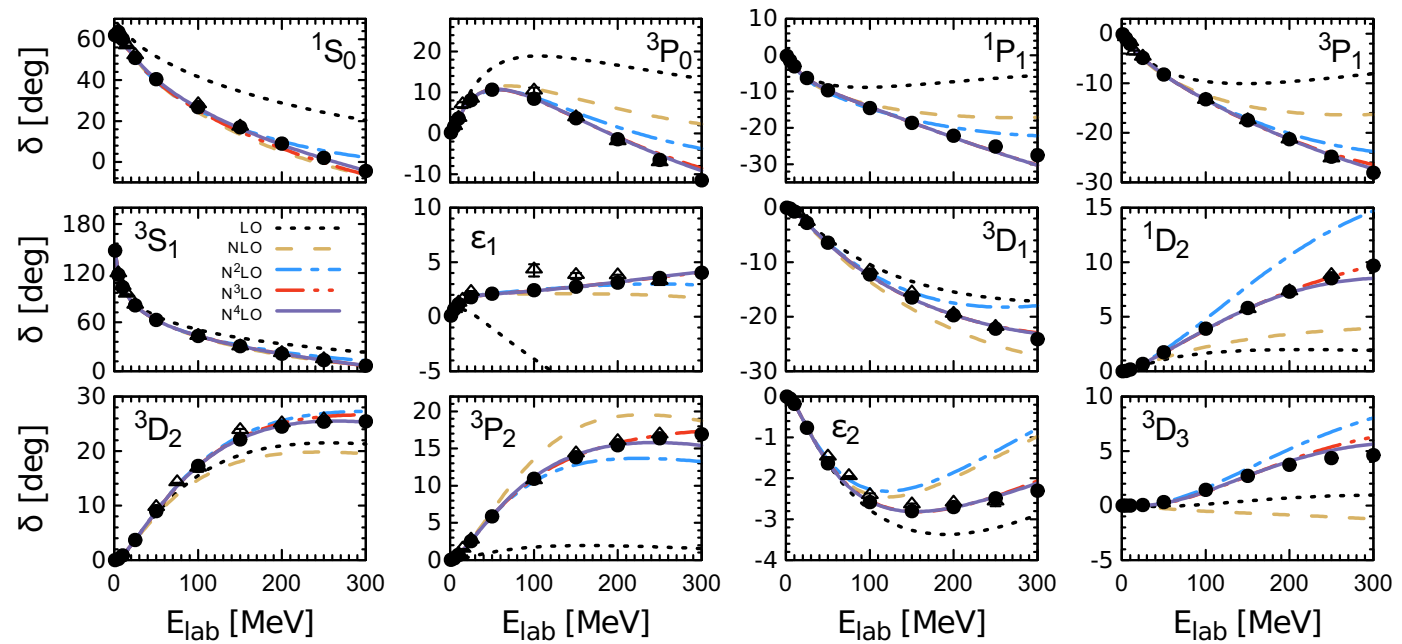

Figure 1. Chiral expansion of the NN phase shifts in comparison with the NPWA (solid dots) and the GWU single-energy np partial wave analysis (open triangles). Dotted (black), dashed (brown), dash-dotted (blue), dashdouble-dotted (red) and solid (violet) lines show the results at LO, NLO, $\mathrm{N}^{2} \mathrm{LO}, \mathrm{N}^{3} \mathrm{LO}$ and $\mathrm{N}^{4} \mathrm{LO}$, respectively, calculated using the cutoff $R=0.9 \mathrm{fm}$

Table 1. $\chi^{2} /$ datum for the description of the Nijmegen np and pp phase shifts [30] at different orders in the chiral expansion for the cutoff $R=0.9 \mathrm{fm}$. The numbers in the brackets give the number of adjustable isospin-invariant contact interactions at the corresponding order. Only those channels are included which have been used in the $\mathrm{N}^{3} \mathrm{LO} / \mathrm{N}^{4} \mathrm{LO}$ fits, namely the S-, P- and D-waves and the mixing angles $\epsilon_{1}$ and $\epsilon_{2}$.

\begin{tabular}{|c|c|c|c|c|c|}
\hline$E_{\text {lab }}$ bin & LO (2) & NLO (9) & $\mathrm{N}^{2} \mathrm{LO}(9)$ & $\mathrm{N}^{3} \mathrm{LO}(24)$ & $\mathrm{N}^{4} \mathrm{LO}(24)$ \\
\hline \multicolumn{6}{|c|}{ neutron-proton phase shifts } \\
\hline $0-100$ & 360 & 31 & 4.5 & 0.7 & 0.3 \\
\hline $0-200$ & 480 & 63 & 21 & 0.7 & 0.3 \\
\hline \multicolumn{6}{|c|}{ proton-proton phase shifts } \\
\hline $0-100$ & 5750 & 102 & 15 & 0.8 & 0.3 \\
\hline $0-200$ & 9150 & 560 & 130 & 0.7 & 0.6 \\
\hline
\end{tabular}

corresponding two-pion exchange components without invoking new parameters. ${ }^{1}$ These results constitute an important consistency check of the theoretical approach and provide a nice illustration of the importance of the chiral $2 \pi$-exchange potential, which is determined entirely by the chiral symmetry of QCD and experimental information on $\pi N$ scattering. Furthermore, the obtained results suggest - fully in line with the Weinberg power counting [3] - that the theoretical uncertainties at NLO and $\mathrm{N}^{3} \mathrm{LO}$ are dominated by the neglected $2 \pi$-exchange contributions at orders $\mathrm{N}^{2} \mathrm{LO}$ and $\mathrm{N}^{4} \mathrm{LO}$, respectively. Indeed, if certain order- $Q^{4}$ and order- $Q^{6}$ contact interactions would have to be promoted to lower orders in violation with naive dimensional analysis, see e.g. [32-34], the inclusion of the $2 \pi$ -

\footnotetext{
${ }^{1} \mathrm{At} \mathrm{N}{ }^{4} \mathrm{LO}$, there is one additional isospin-breaking contact interaction in the neutron-proton ${ }^{1} \mathrm{~S}_{0}$ channel.
} 
exchange contributions alone at $\mathrm{N}^{2} \mathrm{LO}$ and $\mathrm{N}^{3} \mathrm{LO}$ would not result in the improved accuracy of the fits.

We have also tested the convergence of the chiral expansion for various observables. For example, for the np total cross section at various energies, we obtain using $R=0.9 \mathrm{fm}$

$$
\begin{aligned}
\sigma_{\text {tot }}(50 \mathrm{MeV}) & =183.6_{\mathrm{LO}}-17.1_{\mathrm{NLO}}+0.5_{\mathrm{N}^{2} \mathrm{LO}}-0.2_{\mathrm{N}^{3} \mathrm{LO}}+0.7_{\mathrm{N}^{4} \mathrm{LO}}=167.5 \mathrm{mb}, \\
\sigma_{\text {tot }}(96 \mathrm{MeV}) & =84.8_{\mathrm{LO}}-9.7_{\mathrm{NLO}}+3.2_{\mathrm{N}^{2} \mathrm{LO}}-0.8_{\mathrm{N}^{3} \mathrm{LO}}+0.5_{\mathrm{N}^{4} \mathrm{LO}}=78.0 \mathrm{mb}, \\
\sigma_{\text {tot }}(143 \mathrm{MeV}) & =52.5_{\mathrm{LO}}-3.4_{\mathrm{NLO}}+5.1_{\mathrm{N}^{2} \mathrm{LO}}-0.5_{\mathrm{N}^{3} \mathrm{LO}}+0.2_{\mathrm{N}^{4} \mathrm{LO}}=53.9 \mathrm{mb}, \\
\sigma_{\text {tot }}(200 \mathrm{MeV}) & =34.9_{\mathrm{LO}}+1.0_{\mathrm{NLO}}+6.7_{\mathrm{N}^{2} \mathrm{LO}}+0.6_{\mathrm{N}^{3} \mathrm{LO}}-0.5_{\mathrm{N}^{4} \mathrm{LO}}=42.7 \mathrm{mb} .
\end{aligned}
$$

Notice that the natural size of the $\mathrm{N}^{2} \mathrm{LO}, \mathrm{N}^{3} \mathrm{LO}$ and $\mathrm{N}^{4} \mathrm{LO}$ contributions at $E=50 \mathrm{MeV}$ is estimated to be $\sim 3 \mathrm{mb}, \sim 0.8 \mathrm{mb}$ and $\sim 0.2 \mathrm{mb}$, respectively [24]. The somewhat large $\mathrm{N}^{4} \mathrm{LO}$ contribution at this energy reflects, in fact, the adopted fitting protocol, see [25] for more details. Similarly, the NLO contributions at the two highest energies come our rather small compared with their expected size of $\sim 10 \mathrm{mb}$. Generally, the convergence pattern of the chiral expansion for the np cross section is very satisfactory. We also found rapid convergence for the deuteron properties [24, 25].

\section{Uncertainty quantification}

Uncertainty quantification is key for performing a meaningful comparison between theory and data. In the past, theoretical uncertainty of nuclear chiral EFT due to truncation of the chiral expansion was, at best, estimated via a variation of the cutoff in a certain range. As pointed out already in [4], such an approach suffers from certain drawbacks. First, the residual cutoff dependence of a calculated observable measures the impact of neglected contact interactions which contribute only at even orders $Q^{2 n}, n=0,1,2, \ldots$. Secondly, the available cutoff range appears, in practice, to be rather limited. As a result, such "cutoff bands" can generally not be expected to provide a realistic estimation of the theoretical uncertainty, see [4, 24, 25] for more detail.

In [24], we proposed an alternative approach to quantify the uncertainty of a chiral EFT prediction by directly estimating the size of neglected higher-order contributions to a given observable. To this aim, we assume the expansion parameter $Q$ to be $Q=\max \left\{M_{\pi} / \Lambda_{b},|\vec{p}| / \Lambda_{b}\right\}$. The breakdown scale of the chiral expansion $\Lambda_{b}$ was estimated in [24] to be $\Lambda_{b} \sim 600 \mathrm{MeV}$ for $R=0.8 \ldots 1.0 \mathrm{fm}, \Lambda_{b} \sim$ $500 \mathrm{MeV}$ for $R=1.1 \mathrm{fm}$ and $\Lambda_{b} \sim 400 \mathrm{MeV}$ for $R=1.2 \mathrm{fm}$. The accuracy of a chiral EFT prediction for the observable of interest can then be estimated by multiplying the leading-order result with the appropriate power of $Q$. In order to have as reliable as possible estimation of the uncertainty, we proposed to also employ the information on the actual size of higher-order contributions (if available). The precise description of the suggested algorithm for error analysis can be found in [24, 25], see also [35] for a related discussion. Here we just mention that our new approach can be straightforwardly applied (i) to any observable of interest and (ii) for any specific choice of the regulator $R$.

As a representative example, consider the chiral EFT predictions for the np total cross section in Eq. (2). Performing the error analysis as described above, our final predictions for $R=0.9 \mathrm{fm}$ at $\mathrm{N}^{4} \mathrm{LO}$ take the form $\sigma_{\text {tot }}(50 \mathrm{MeV})=167.5 \pm 0.2 \mathrm{mb}, \sigma_{\text {tot }}(96 \mathrm{MeV})=78.0 \pm 0.2 \mathrm{mb}, \sigma_{\text {tot }}(143 \mathrm{MeV})=$ $53.9 \pm 0.4 \mathrm{mb}$ and $\sigma_{\text {tot }}(200 \mathrm{MeV})=42.7 \pm 0.9 \mathrm{mb}$. These predictions are in excellent agreement with the NPWA results of $\sigma_{\mathrm{NPWA}}(50 \mathrm{MeV})=167.8 \pm 0.2 \mathrm{mb}, \sigma_{\mathrm{NPWA}}(96 \mathrm{MeV})=78.1 \pm 0.5 \mathrm{mb}$, $\sigma_{\mathrm{NPWA}}(143 \mathrm{MeV})=53.6 \pm 0.5 \mathrm{mb}$ and $\sigma_{\mathrm{NPWA}}(200 \mathrm{MeV})=42.35 \pm 0.20 \mathrm{mb}$. Last but not least, our quoted theoretical uncertainties were found in [36] to be consistent with the $68 \%$ degree-of-belief intervals for EFT predictions. 

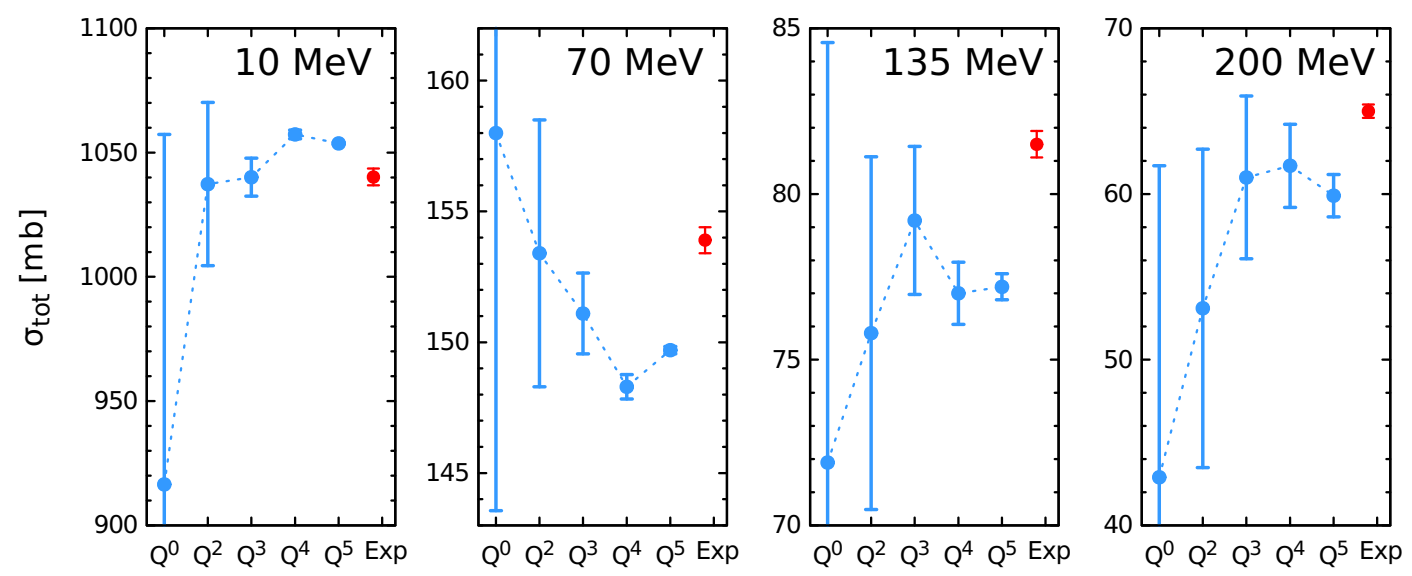

Figure 2. Predictions for $\mathrm{Nd}$ total cross section at laboratory energies of 10, 70, 135 and $200 \mathrm{MeV}$ based on the $\mathrm{NN}$ potentials of $[24,25]$ for $R=0.9 \mathrm{fm}$ without including the $3 \mathrm{NF}$.

\section{Beyond the NN system}

With all the necessary tools being developed as described in the previous sections, one is now well equipped for testing chiral EFT and especially the novel chiral 3NFs in three- and more-nucleon systems. As a first step along this line, the new generation of the chiral NN potentials has been recently applied to nucleon-deuteron scattering and selected low-energy observables in ${ }^{3} \mathrm{H},{ }^{4} \mathrm{He}$ and ${ }^{4} \mathrm{Li}$ [35]. With a detailed account of this study being given in the talks by Maris [37] and Skibinski [38], I only briefly address here the results for nucleon-deuteron $(\mathrm{Nd})$ total cross section visualized in Fig. 2. While these calculations can, starting from $\mathrm{N}^{2} \mathrm{LO}$, not be regarded as complete due to the missing $3 \mathrm{NFs}$, they do reveal a number of interesting insights. First, one observes a convergent pattern at all considered energies in spite of the fact that the calculations are actually incomplete. The predicted results for the cross section at $\mathrm{N}^{4} \mathrm{LO}$ are in a clear disagreement with experimental values with the discrepancies being much larger than the experimental and estimated theoretical uncertainties. This provides truly unambiguous evidence for missing $3 \mathrm{NF}$ within the employed theoretical framework. The estimated accuracy of the chiral EFT results at $\mathrm{N}^{4} \mathrm{LO}$ suggests that Nd scattering at intermediate and even higher energies will be a very promising testing ground for the chiral 3NF. Furthermore, it is comforting to see that the deviations between our $\mathrm{N}^{3,4} \mathrm{LO}$ predictions and experimental data are similar in size to the NLO (i.e. $Q^{2}$ ) error bars, which give the estimated size of $\mathrm{N}^{2} \mathrm{LO}$ contributions. Indeed, this is precisely the chiral order at which the first nonvanishing $3 \mathrm{NF}$ appear according to the Weinberg power counting.

To summarize, the newly developed improved chiral NN potentials up to $\mathrm{N}^{4} \mathrm{LO}$, along with a new analysis of the theoretical uncertainties, open the way for precision tests of chiral $3 \mathrm{NFs}$ in few-nucleon systems. Work along these lines is in progress by the LENPIC collaboration [39].

\section{Acknowledgements}

I am grateful to my collaborators Hermann Krebs, Ulf-G. Meißner and to all members of the LENPIC collaboration for sharing their insights into the discussed topics. This work was supported in part by DFG (SFB/TR 16, "Subnuclear Structure of Matter") and ERC project 259218 NUCLEAREFT. 


\section{References}

[1] E. Epelbaum, H. W. Hammer and U.-G. Meißner, Rev. Mod. Phys. 81, 1773 (2009).

[2] R. Machleidt and D. R. Entem, Phys. Rept. 503, 1 (2011).

[3] S. Weinberg, Phys. Lett. B 251, 288 (1990).

[4] E. Epelbaum, W. Glockle and U.-G. Meißner, Nucl. Phys. A 747, 362 (2005).

[5] D. R. Entem and R. Machleidt, Phys. Rev. C 68, 041001 (2003).

[6] V. Bernard, E. Epelbaum, H. Krebs and U.-G. Meißner, Phys. Rev. C 77, 064004 (2008).

[7] V. Bernard, E. Epelbaum, H. Krebs and U.-G. Meißner, Phys. Rev. C 84, 054001 (2011).

[8] E. Epelbaum, Eur. Phys. J. A 34, 197 (2007).

[9] S. Kölling, E. Epelbaum, H. Krebs and U.-G. Meißner, Phys. Rev. C 80, 045502 (2009).

[10] S. Kölling, E. Epelbaum, H. Krebs and U.-G. Meißner, Phys. Rev. C 84, 054008 (2011).

[11] S. Pastore, R. Schiavilla and J. L. Goity, Phys. Rev. C 78, 064002 (2008).

[12] S. Pastore et al., Phys. Rev. C 80, 034004 (2009).

[13] J. Golak et al., Eur. Phys. J. A 43, 241 (2010).

[14] K. Hebeler et al., Phys. Rev. C 91, no. 4, 044001 (2015).

[15] H. W. Hammer, A. Nogga and A. Schwenk, Rev. Mod. Phys. 85, 197 (2013).

[16] D. Lee, these proceedings.

[17] N. Kalantar-Nayestanaki et al., Rept. Prog. Phys. 75, 016301 (2012).

[18] R. Machleidt and D. R. Entem, J. Phys. G 37, 064041 (2010).

[19] H. Krebs, A. Gasparyan and E. Epelbaum, Phys. Rev. C 85, 054006 (2012).

[20] H. Krebs, A. Gasparyan and E. Epelbaum, Phys. Rev. C 87, no. 5, 054007 (2013).

[21] E. Epelbaum, A. M. Gasparyan, H. Krebs and C. Schat, Eur. Phys. J. A 51, no. 3, 26 (2015).

[22] R. J. Furnstahl, D. R. Phillips and S. Wesolowski, J. Phys. G 42, no. 3, 034028 (2015).

[23] D. R. Entem, N. Kaiser, R. Machleidt and Y. Nosyk, Phys. Rev. C 91, no. 1, 014002 (2015).

[24] E. Epelbaum, H. Krebs and U.-G. Meißner, Eur. Phys. J. A 51, no. 5, 53 (2015).

[25] E. Epelbaum, H. Krebs and U.-G. Meißner, arXiv:1412.4623 [nucl-th].

[26] V. Baru et al., Eur. Phys. J. A 48, 69 (2012).

[27] E. Epelbaum, W. Glöckle and U.-G. Meißner, Eur. Phys. J. A 19, 125 (2004).

[28] M. Hoferichter, J. R. de Elvira, B. Kubis and U.-G. Meißner, arXiv:1507.07552 [nucl-th].

[29] K. A. Wendt, B. D. Carlsson and A. Ekström, arXiv:1410.0646 [nucl-th].

[30] V. G. J. Stoks et al., Phys. Rev. C 48, 792 (1993).

[31] V. G. J. Stoks et al., Phys. Rev. C 49, 2950 (1994).

[32] A. Nogga, R. G. E. Timmermans and U. van Kolck, Phys. Rev. C 72, 054006 (2005).

[33] M. C. Birse, PoS CD 09, 078 (2009).

[34] B. Long and C. J. Yang, Phys. Rev. C 85, 034002 (2012).

[35] S. Binder et al., arXiv:1505.07218 [nucl-th].

[36] R. J. Furnstahl et al., Phys. Rev. C 92, no. 2, 024005 (2015).

[37] P. Maris, these proceedings.

[38] R. Skibinski, these proceedings.

[39] Low Energy Nuclear Physics International Collaboration (LENPIC), www.lenpic.org. 